\title{
Recent CMS results in conventional and exotic hadron spectroscopy
}

\author{
Vincenzo Mastrapasqua ${ }^{a, \dagger, *}$ \\ ${ }^{a}$ Dipartimento Interateneo di Fisica, Università degli Studi di Bari "Aldo Moro", \\ via Giovanni Amendola n.173, 70126 - Bari, Italy \\ E-mail: vincenzo.mastrapasqua@cern.ch
}

The present report summarizes recent CMS results in conventional and exotic hadron spectroscopy, obtained using the data collected at the Large Hadron Collider during the Run-2 data taking (2015-2018) either with proton-proton collisions at $\sqrt{s}=13 \mathrm{TeV}$ or lead-lead collisions at $\sqrt{s_{N N}}$ $=5.02 \mathrm{TeV}$ per nucleon pair. The results include the first observation of the $B_{s}^{0} \rightarrow X(3872) \phi$ decay mode, evidence of $\mathrm{X}(3872)$ production in lead-lead collisions, a search for intermediate resonances in the $B^{0} \rightarrow \psi(2 S) K_{s}^{0} \pi^{+} \pi^{-}$multi-body decay, and the observation of $\Lambda_{b}^{* *}$ and $\Xi_{b}^{* * *}$ excited states.

*** Particles and Nuclei International Conference - PANIC2021 ***

*** 5 - 10 September, $2021 * * *$

$* * *$ Online $* * *$

\footnotetext{
*Speaker

$\dagger$ On behalf of the CMS Collaboration
} 
The heavy flavour production cross section at the Large Hadron Collider (LHC) is several orders of magnitude greater than at $e^{+} e^{-}$colliders. The CMS experiment [1] at LHC exploits its $4 \pi$ coverage and high resolution to perform challenging measurements in the Heavy Flavor sector, despite the complex initial state and high background in environments such as proton-proton $(p p)$ and lead-lead $(\mathrm{PbPb})$ collisions. Some of the recent CMS measurements concerning both conventional and exotic spectroscopy in the charm and beauty sectors are presented here.

\section{Recent CMS results on B meson spectroscopy}

$\mathbf{X}(\mathbf{3 8 7 2})$ production in weak decays from beauty mesons: The observed spectrum of $c \bar{c}$ states below the $D \bar{D}$ threshold agrees well with theoretical predictions [2, 3]. The Belle Collaboration observed the $\mathrm{X}(3872)$ state for the first time in 2003 [4], a resonance above the $D \bar{D}$ threshold but with a very small natural width, that does not fit in the predicted mass spectrum.

CMS reported the first observation of the $B_{s}^{0} \rightarrow X(3872) \phi$ decay, with $X(3872) \rightarrow J / \psi \pi^{+} \pi^{-}$ and $\phi \rightarrow K^{+} K^{-}$[5]. The analysis is performed using $p p$ collision data recorded by CMS during the LHC Run-2 in 2016-2018 at a centre-of-mass energy of $13 \mathrm{TeV}$, corresponding to an integrated luminosity of $140 \mathrm{fb}^{-1}$. The analysis uses the decay $B_{s}^{0} \rightarrow \psi(2 S) \phi$ in the same final state as normalisation channel. The ratio $R$ is measured:

$$
R=\frac{\mathcal{B}\left(B_{s}^{0} \rightarrow X(3872) \phi\right) \cdot \mathcal{B}\left(X(3872) \rightarrow J / \psi \pi^{+} \pi^{-}\right)}{\mathcal{B}\left(B_{s}^{0} \rightarrow \psi(2 S) \phi\right) \cdot \mathcal{B}\left(\psi(2 S) \rightarrow J / \psi \pi^{+} \pi^{-}\right)}=\frac{N\left[B_{s}^{0} \rightarrow X(3872) \phi\right] \cdot \varepsilon_{B_{s}^{0} \rightarrow \psi(2 S) \phi}}{N\left[B_{s}^{0} \rightarrow \psi(2 S) \phi\right] \cdot \varepsilon_{B_{s}^{0} \rightarrow X(3872) \phi}}
$$

where $N$ and $\varepsilon$ are respectively the signal yield and the overall efficiency for the decay channel. The nearly identical kinematics of the decay topology of the signal and normalisation channels leads to the cancellation of many systematic uncertainties in the ratio.

The signal yields are separately extracted for the two decay channels using a 2D unbinned maximum likelihood (UML) fit, while the efficiencies are estimated by means of Monte Carlo (MC) techniques. The measured ratio is $R=[2.21 \pm 0.29$ (stat.) \pm 0.17 (syst.) $] \%$, which is consistent with the measurement published by the LHCb Collaboration [6] in 2021.

Since the properties of the decay involving the $\psi(2 S)$ are well-known, it is found that $\mathcal{B}\left[B_{s}^{0} \rightarrow X(3872) \phi\right] \mathcal{B}\left[X(3872) \rightarrow J / \psi \pi^{+} \pi^{-}\right]=(4.14 \pm 0.54$ (stat.) \pm 0.32 (syst.) $\pm 0.46(\mathcal{B})) 10^{-6}$. This can be compared to the analogous branching fraction products in $B^{0}$ and $B^{+}$decays: the measured value for $B_{s}^{0}$ is consistent with the $B^{0}$ result, but it is about two times smaller than the one for $B^{+}$. Furthermore, the ratio for $B_{s}^{0}$ and $B^{+}$results for the $X(3872)$ is significantly lower than the corresponding one for the decays involving the $\psi(2 S)$. An explanation of the observed difference has been proposed within the tetraquark model of the X(3872) state [7].

First evidence of $\mathbf{X}(\mathbf{3 8 7 2})$ production in $\boldsymbol{P b P b}$ collisions: Information on the $\mathrm{X}(3872)$ state can be obtained from studying its production and survival in a quark-gluon plasma [8, 9]. The $\mathrm{X}(3872)$ production has been studied in $\mathrm{PbPb}$ collisions at $\sqrt{s_{N N}}=5.02 \mathrm{TeV}$ per nucleon pair, using the decay chain $X(3872) \rightarrow J / \psi\left(\rightarrow \mu^{+} \mu^{-}\right) \pi^{+} \pi^{-}$[10]. The analysis uses $1.7 \mathrm{nb}^{-1}$ of data collected by the CMS detector in 2018. The measurement is performed in the rapidity and transverse momentum ranges $|\mathrm{y}|<1.6$ and $15<p_{T}<50 \mathrm{GeV} / \mathrm{c}$. 
Figure 1: The prompt $\mathrm{X}(3872)$ over $\psi(2 S)$ yields ratio $\rho^{P b P b}$ in $P b P b$ collisions at $\sqrt{s_{N N}}=5.02 \mathrm{TeV}$ [10]. The vertical bars (boxes) correspond to statistical (systematic) uncertainties. The yield ratios $\rho^{p p}$ in $p p$ collisions at $\sqrt{s}=8 \mathrm{TeV}$, measured by ATLAS [11], and at $\sqrt{s}=7 \mathrm{TeV}$, measured by CMS [12] are shown.

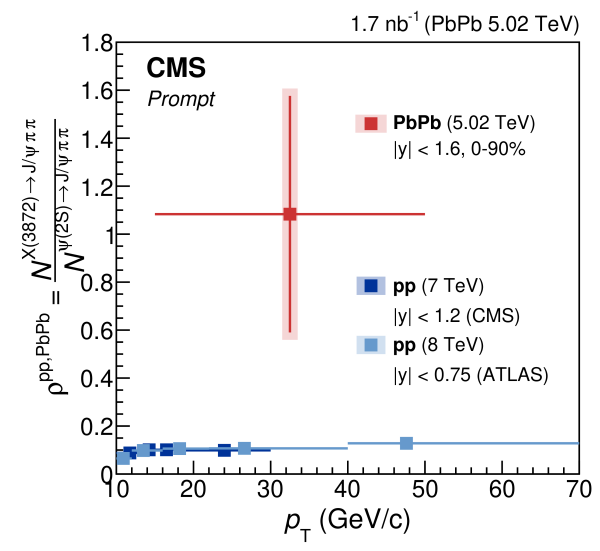

The analysis focuses on promptly-produced X(3872), from charm quark fragmentation. The $\psi(2 S)$ decay in the same final state is considered as control channel. The candidates are reconstructed and selected inclusively, i.e. with no requirement on the production mechanism, then the signal yields are extracted with an UML fit. Finally the inclusive yields are corrected to consider the overall efficiency, estimated with MC simulations, and to factor out the non-prompt contribution coming from b-hadrons, estimated on data. The ratio $\rho^{i}(\mathrm{i}=p p, P b P b)$ between the corrected signal yields for $\mathrm{X}(3872)$ and $\psi(2 S)$ is measured (Fig. 1); with the available level of statistics, $\rho^{P b P b}$ is compatible with both 1 , within $1 \sigma$, and $\rho^{p p} \simeq 0$. in Run-3 at the LHC will improve the measurement, thus leading to further understanding of the internal structure of X(3872) and its production mechanism.

Intermediate resonances in B meson decays: CMS also recently reported the two decays $B^{0} \rightarrow \psi(2 S) K_{s}^{0} \pi^{+} \pi^{-}$and $B_{s}^{0} \rightarrow \psi(2 S) K_{s}^{0}$, observed using a data sample of $103.7 \mathrm{fb}^{-1}$ of $p p$ collisions collected at $\sqrt{s}=13 \mathrm{TeV}$ in 2017 and 2018 [13].

The multi-body decay $B^{0} \rightarrow \psi(2 S) K_{s}^{0} \pi^{+} \pi^{-}$allows the search for intermediate exotic resonances. The two-body and three-body invariant mass distributions of the $B^{0}$ decay products are investigated and do not show any significant exotic narrow structure in addition to the already known light meson resonances $\left(\rho(770), K^{*}(892)^{ \pm}, K_{1}(1270)^{0}\right)$ with the current sample size.

\section{Recent CMS results on baryon spectroscopy}

Observation of excited $\Lambda_{b}$ states: A study of the $\Lambda_{b}^{0} \pi^{+} \pi^{-}$invariant mass distribution in the 5.9-6.4 GeV range is performed using up to $140 \mathrm{fb}^{-1}$ of $p p$ collisions data at $\sqrt{s}=13 \mathrm{TeV}$ collected by CMS during the 2016-2018 period [14]. The $\Lambda_{b}^{0}$ candidates are reconstructed in three different channels separately: (1) $\Lambda_{b}^{0} \rightarrow J / \psi\left(\rightarrow \mu^{+} \mu^{-}\right) \Lambda^{0}$, (2) $\Lambda_{b}^{0} \rightarrow \psi(2 S)\left(\rightarrow \mu^{+} \mu^{-}\right) \Lambda^{0}$, and (3) $\Lambda_{b}^{0} \rightarrow \psi(2 S)\left(\rightarrow \mu^{+} \mu^{-} \pi^{+} \pi^{-}\right) \Lambda^{0}$, with $\Lambda^{0} \rightarrow p \pi^{-}$.

The $\Lambda_{b}^{0} \pi^{+} \pi^{-}$candidates are then built by adding two opposite-sign tracks to the $\Lambda_{b}^{0}$ candidate, while same-sign track pairs are used to define the control region. Further selection is applied, separately optimized for the two regions $m_{\Lambda_{b}^{0} \pi^{+} \pi^{-}} \lessgtr 5.95 \mathrm{GeV}$. Two signals corresponding to the excitations $\Lambda_{b}(5912)$ and $\Lambda_{b}(5920)$, already observed by LHCb [15] and CDF [16], are observed near the kinematic threshold with significance of $5.7 \sigma$ and well over $6 \sigma$, respectively (Fig. 2, left). 

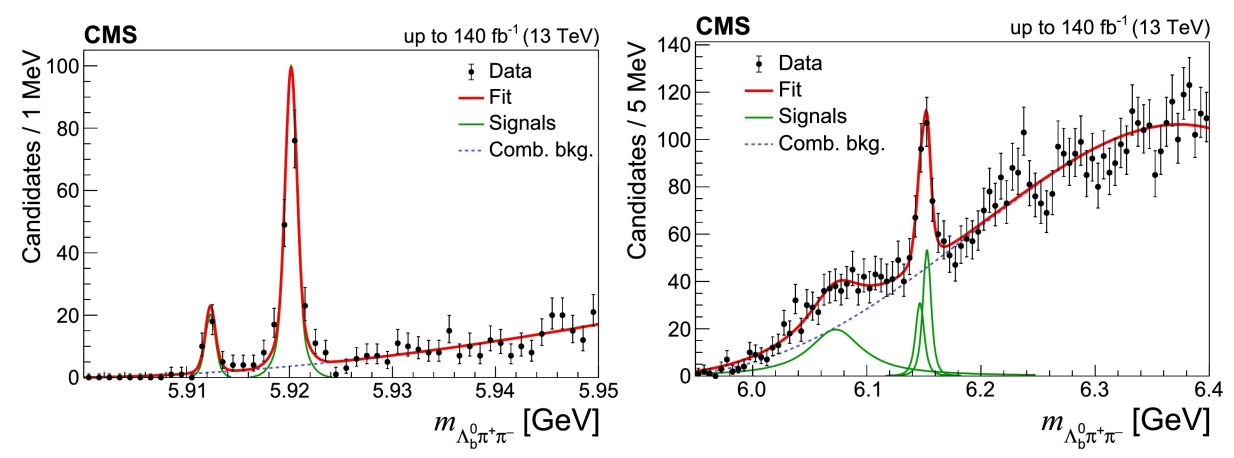

Figure 2: Invariant mass distribution of the selected $\Lambda_{b}^{0} \pi^{+} \pi^{-}$candidates near the kinematic threshold (left) and in the high-mass region (right) [14]. Four signals are considered corresponding to the known $\Lambda_{b}$ excitations: $\Lambda_{b}(5912), \Lambda_{b}(5920), \Lambda_{b}(6146), \Lambda_{b}(6152)$. An additional contribution is introduced to describe the broad enhancement observed in the region below $6.1 \mathrm{GeV}$.

In the high-mass region (Fig. 2, right) a narrow peak at $6150 \mathrm{MeV}$ with a resolution of $3.8 \mathrm{MeV}$ is observed, consistent with the superposition of the two $\Lambda_{b}$ excited states $\Lambda_{b}(6146)$ and $\Lambda_{b}(6152)$, already observed at $\mathrm{LHCb}$ [17]. There is evidence of a broad enhancement in the region below $6.1 \mathrm{GeV}$, not present in the control region. A veto on possible contributions from intermediate states $\left(\Sigma_{b}^{(*) \pm} \rightarrow \Lambda_{b}^{0} \pi^{ \pm}\right)$improves the agreement between the signal and control regions, but this hypothesis cannot be tested with the available sample size. A similar structure has been later observed at LHCb and it has been interpreted as a further excited state, $\Lambda_{b}^{0}(6072)$ [18].

Observation of a new excited $\Xi_{b}$ state: CMS has also reported the observation of a new state in the $\Xi_{b}^{-} \pi^{+} \pi^{-}$system using up to $140 \mathrm{fb}^{-1}$ of $p p$ collisions data at $\sqrt{s}=13 \mathrm{TeV}$ collected during the 2016-2018 period at LHC [19]. The event selection requires a combination of dimuon triggers targeting $J / \psi \rightarrow \mu^{+} \mu^{-}$, then $\Xi_{b}^{-}$candidates are reconstructed in three decay channels separately: (1) $\Xi_{b}^{-} \rightarrow J / \psi \Xi^{-}$, (2) $\Xi_{b}^{-} \rightarrow J / \psi \Lambda^{0} K^{-}$and (3) $\Xi_{b}^{-} \rightarrow J / \psi \Sigma^{0} K^{-}$, with $\Xi^{-} \rightarrow \Lambda^{0} \pi^{-}, \Lambda^{0} \rightarrow p \pi^{-}$ and $\Sigma^{0} \rightarrow \Lambda^{0} \gamma_{\text {soft }}$, where the soft photon $\gamma_{\text {soft }}$ is not reconstructed. The candidates are selected with criteria optimized for each decay channel and the signal yields are extracted with an UML fit.

Excited $\Xi_{b}^{-}$candidates are reconstructed by adding two opposite-sign tracks from the same $p p$ collision vertex as $\Xi_{b}^{-}$to it, while same-sign tracks are used to define the control region. Since the contribution of the intermediate resonance $\Xi_{b}^{* 0} \rightarrow \Xi_{b}^{-} \pi^{+}$is expected to be dominant [20, 21], the requirement $m\left(\Xi_{b}^{* 0}\right)-m\left(\Xi_{b}^{-}\right)-m_{\pi}^{P D G}<20.73 \mathrm{MeV}$ is added (peak expected at $15.73 \mathrm{MeV}$ ). The fully reconstructed channels (1) and (2) are combined as they have similar resolution.

A simultaneous UML fit is performed on the two data samples, which results in the observation of a narrow peak at $m\left(\Xi_{b}^{* *-}\right)=6100.3 \pm 0.2$ (stat.) \pm 0.1 (syst.) $\pm 0.6\left(\Xi_{b}^{-}\right) \mathrm{MeV}$, where the last term originates from the uncertainties on the $\Xi_{b}^{-}$mass, with local statistic significance greater than $6 \sigma$. An upper limit on the resonance width is set at $95 \%$ confidence level: $\Gamma\left(\Xi_{b}^{* *-}\right)<1.9 \mathrm{MeV}$. Since the new $\Xi_{b}(6100)^{-}$is consistent with the lightest orbitally excited baryon, the analogy with the $\Xi_{c}$ system $[20,21]$ suggests its spin and light diquark angular momentum are $J^{P}=3 / 2^{-}$and $j_{d s}=1$.

\section{References}

[1] CMS Collaboration, The CMS experiment at the CERN LHC, JINST 3 S08004 (2008) 
[2] M. B. Voloshin, Charmonium, Prog. Part. Nucl. Phys. 61455 (2008)

[3] N. Brambilla et al., Heavy quarkonium: progress, puzzles and opportunities, Eur. Phys. J. C., 711534 (2011)

[4] Belle Collaboration, Observation of a narrow charmoniumlike state in exclusive $B^{ \pm} \rightarrow$ $K^{ \pm} \pi^{+} \pi^{-} J / \psi$, Phys. Rev. Lett. 91, 262001 (2003)

[5] CMS Collaboration, Observation of the $B_{s}^{0} \rightarrow X(3872) \phi$ decay, Phys. Rev. Lett. 125, 152001 (2020)

[6] LHCb Collaboration, Study of $B_{s}^{0} \rightarrow J / \psi \pi^{+} \pi^{-} K^{+} K^{-}$decays, JHEP 02, 024 (2021)

[7] L. Maiani et al., X(3872) tetraquarks in B and Bs decays, Phys. Rev. D. 102034017 (2020)

[8] H. Zhang et al., Deciphering the nature of X(3872) in Heavy Ion Collisions, Phys. Rev. Lett. 126012301 (2021)

[9] B. Wu et al., X(3872) Transport in Heavy-Ion Collisions, Eur. Phys. J. A 57, 122 (2021)

[10] CMS Collaboration, Evidence for X(3872) in PbPb collisions and studies of its prompt production at $\sqrt{s_{N N}}=5.02 \mathrm{TeV}$, https://arxiv.org/abs/2102.13048, submitted to Phys. Rev. Lett.

[11] ATLAS Collaboration, Measurements of $\psi(2 S)$ and $X(3872) \rightarrow J / \psi \pi^{+} \pi^{-}$production in $p p$ collisions at $\sqrt{s}=8 \mathrm{TeV}$ with the ATLAS detector, JHEP 01117 (2017)

[12] CMS Collaboration, Measurement of the X(3872) production cross section via decays to $J / \psi \pi^{+} \pi^{-}$in pp collisions at $\sqrt{s}=7 \mathrm{TeV}$, JHEP 04154 (2013)

[13] CMS Collaboration, Observation of $B^{0} \rightarrow \psi(2 S) K_{s}^{0} \pi^{+} \pi^{-}$and $B_{s}^{0} \rightarrow \psi(2 S) K_{s}^{0}$ decays, CMSPAS-BPH-18-004

[14] CMS Collaboration, Study of excited $\Lambda_{b}^{0}$ states decaying to $\Lambda_{b}^{0} \pi^{+} \pi^{-}$in proton-proton collisions at $\sqrt{s}=13 \mathrm{TeV}$, Phys. Lett. B 803, 135345 (2020)

[15] LHCb Collaboration, Observation of excited $\Lambda_{b}^{0}$ baryons, Phys. Rev. Lett. 109, 172003 (2012)

[16] CDF Collaboration, Evidence for a bottom baryon resonance $\Lambda_{b}^{* 0}$ in CDF data, Phys. Rev. D. 88, 071101 (2013)

[17] LHCb Collaboration, Observation of New Resonances in the $\Lambda_{b}^{0} \pi^{+} \pi^{-}$System, Phys. Rev. Lett. 123, 152001 (2019)

[18] LHCb Collaboration, Observation of a new baryon state in the $\Lambda_{b}^{0} \pi^{+} \pi^{-}$mass spectrum, JHEP 06, 136 (2020)

[19] CMS Collaboration, Observation of a New Excited Beauty Strange Baryon Decaying to $\Xi_{b}^{-} \pi^{+} \pi^{-}$, Phys. Rev. Lett. 126, 252003 (2021)

[20] Particle Data Group, Review of Particle Physics, Prog. Theor. Exp. Phys. 2020, 083 C01 (2020)

[21] CLEO Collaboration, Evidence of New States Decaying into $\Xi_{c}^{*} \pi$, Phys. Rev. Lett. 83, 3390 (1999) 